\title{
Research on Intelligent Transportation System Based on Improved Ant Colony Algorithm
}

\author{
Xingli Wu, Huimin $\mathrm{Lv}^{+}$and Shigang Cui \\ Tianjin University of Technology and Education, Tianjin Key Laboratory of Information Sensing \& \\ Intelligent Control, Tianjin, China
}

\begin{abstract}
At present, the optimal path planning based on the path condition has become one of the key research directions of the intelligent transportation system. Based on the characteristics and existing problems of the actual intelligent transportation system, this paper studied the intelligent transportation system based on improved ant colony algorithm. Firstly, we used the Dijkstra algorithm to plan an initial sub-optimal path, and then established the objective function by combining the distance, time and cost of vehicle travel, and improved pheromone update method. Finally using the improved ant colony algorithm simulation results, the effectiveness of the proposed method was verified. The experimental results show that the improved ant colony algorithm is used to plan the path in the intelligent transportation system and finally solve the optimal driving route. It has been improved in terms of efficiency and effectiveness, which can basically meet customer requirements and make full use of road resources and vehicle resources.
\end{abstract}

Keywords: ant colony algorithm, intelligent transportation system, path planning, Dijkstra algorithm

\section{Introduction}

Intelligent transportation system is a service system that combines electronic information technology with the traditional transportation industry. It is based on information technology, control technology and other technologies. With the development of new generation of electronic information technology, it provides an efficient and adaptable modern transportation management system [1]. With the rapid growth of population and the continuous improvement of people's material living standards, driving alone has become the main mode of transportation for people to travel. If the intelligent transportation system can provide the driver with an optimal travel route according to the actual situation, such as distance, time and cost, path planning for vehicles, it will reduce road congestion, traffic accidents and waste of resources, and improve road utilization.

For the current problems in the intelligent transportation system, a large number of scholars at home and abroad have conducted research on this issue. They use Dijkstra algorithm, genetic algorithm, neural network algorithm and ant colony algorithm to plan vehicle path, so as to find an optimal driving path. In the literature [2], based on the genetic algorithm, the minimum cost is used as the objective function, and the optimal scheduling scheme is obtained through genetic operations such as selection, crossover and mutation. In the literature [3], an ant colony algorithm with real-time planning is proposed, and the author adds a local search optimization algorithm for specific problems in the search process, which can realize real-time path induction of vehicles. In the literature [4], by analyzing and transforming traffic constraints, an improved ant colony algorithm is proposed to improve the path planning efficiency of the algorithm. The literature [5] uses Dijkstra-ant colony algorithm to study the path planning of parking system, and combines Dijkstra algorithm with ant colony algorithm to effectively improve the efficiency of path search and improve the quality of search path.

\footnotetext{
${ }^{+}$Corresponding author. Tel.:13920217350.
}

E-mail address:1vhm0403@163.com. 
In this paper, we proposed to generate the initial sub optimal path through the Dijkstra algorithm, and then according to the impact of vehicle travel distance, time and cost on the path selection, finally we used the improved ant colony algorithm to get the overall optimal route. It makes the planning path meet the requirements of the customer, and makes full use of road resources and vehicle resources.

\section{Basic Ant Colony Algorithm}

Ant colony algorithm is a bionic optimization algorithm to simulate foraging behavior of ant colony. Ants can leave a pheromone in the path they are passing through, and they can sense the existence and intensity of this substance. This substance can guide their movement and ants will move to the direction of high concentration of pheromones [6]. The ant colony foraging behavior is a positive feedback phenomenon. Through the positive feedback mechanism, the ant colony can quickly find the shortest path between the nest and the food source with the change of environment.

There are $m$ ants randomly distributed in $n$ cities. $b_{i}(t)(i=1,2,3, \cdots, n)$ is the number of ants in city $i$ at time $t$, and $m=\sum_{i=1}^{n} b i(t)$. At time $t$, the probability that ant $k$ in the city $i$ chooses to move to the city $j$ can be expressed in formula (1).

$$
p_{i j}^{k}=\left\{\begin{array}{ccc}
\frac{\tau_{i j}^{\alpha}(t) \eta_{i j}^{\beta}(t)}{\sum_{\text {sealowed }_{k} \tau_{i s}^{\alpha}(t) \eta_{i s}^{\beta}(t)}}, \quad j \in \text { allowed }_{k} \\
0 \quad, \quad j \notin \text { allowed }_{k}
\end{array}\right.
$$

Among them, allowed ${ }_{k}$ represents the set of cities that allow ant $\mathrm{k}$ to be allowed to go next; $\tau_{\mathrm{ij}}^{\alpha}$ represents the pheromone factor on $e_{i j} \cdot \eta_{i j}^{\beta}$ represents the visibility factor between city $i$ and $j$, generally taken $\eta_{i j}=1 / d_{i j}\left(d_{i j}\right.$ indicates the distance between the city $\mathrm{i}$ and the city $\mathrm{j}$ ); $\alpha, \beta$ these two parameters reflect the accumulative pheromone and urban illuminating information on the edge of the city during the transfer process. At $t$ time, the ant $\mathrm{k}$ in the city $\mathrm{i}$ decides to transfer to city $\mathrm{j}$. At $\mathrm{t}+1$ time, the ant $\mathrm{k}$ is transferred to the city $\mathrm{j}$, and the amount of pheromone is $\Delta \tau_{\mathrm{ij}}(\mathrm{t}, \mathrm{t}+1)$ on the side $\mathrm{e}_{\mathrm{ij}}$. The pheromone of the side $\mathrm{e}_{\mathrm{ij}}$ corresponding to the road between any two cities i,j can be represented by formula (2).

$$
\left\{\begin{array}{l}
\tau_{i j}(t, t+1)=\rho \tau_{i j}(t)+\Delta \tau_{i j}(t, t+1) \\
\Delta \tau_{i j}(t, t+1)=\sum_{k=1}^{m} \Delta \tau_{i j}^{k}(t, t+1)
\end{array}\right.
$$

In the formula, $\Delta \tau_{\mathrm{ij}}(\mathrm{t}, \mathrm{t}+1)$ is an incremental contribution to the pheromone of the side $\mathrm{e}_{\mathrm{ij}}$ by all the ants in the side $\mathrm{e}_{\mathrm{ij}} . \rho$ (Usually set $0<\rho<1$ ) is the residual coefficient of pheromone, avoiding the infinite accumulation of pheromone of side eij. Generally, $\Delta \tau_{\mathrm{ij}}$ can be generated using ant-density model, antquantity model and ant-week model. Because the incremental information in the ant-week model takes into account the global changes, it ensures that the residual information does not accumulate indefinitely, and non-optimal paths are gradually forgotten over time. Therefore, the ant-week model is used to generate $\Delta \tau_{\mathrm{ij}}$. The ant-week model is shown in formula (3).

$$
\Delta \tau_{\mathrm{ij}}^{\mathrm{k}}(\mathrm{t}, \mathrm{t}+1)=\left\{\begin{array}{cl}
\mathrm{Q} / \mathrm{L}_{\mathrm{k}}, & \text { if ant } \mathrm{k} \text { arrives to city } \mathrm{j} \text { from city } \mathrm{i} \text { in this cycle } \\
0, & \text { otherwise }
\end{array}\right.
$$

$\mathrm{L}_{\mathrm{k}}$ is the length of the path of ant $\mathrm{k}$ in this cycle.

\section{Generate Initial Path}

This paper uses Dijkstra algorithm to generate the initial sub-optimal path. This algorithm is used to calculate the shortest path from one node to all other nodes in the non-negative weight graph. The basic idea is to put the weighted value of all the nodes are divided into two groups. The first group includes the nodes that have determined the shortest path, and the second group is the undetermined shortest path node. According to the order of the shortest path length increasing, second groups of nodes are added to the first group one by one, until all the nodes that are reachable from the source point are included in the first group [7]. 
When using Dijkstra algorithm to solve the optimal planning path, we should first use the MAKLINK graph theory algorithm to establish the two-dimensional space of path planning, and then use Dijkstra algorithm to form a suboptimal path through the path node $S$ (the starting point), $P_{1}, P_{2}, \cdots, P_{d}, T$ (the end point) on the MAKLINK graph. The node corresponds to the free connection is $L_{i}(i=1,2,3, \cdots, d)$, Let $P_{i}^{(0)}$ and $P_{i}^{(1)}$ be the two endpoints of $\mathrm{L}_{\mathrm{i}}$, and the other points on the link can be expressed by formula (4).

$$
\mathrm{P}_{\mathrm{i}}\left(\mathrm{h}_{\mathrm{i}}\right)=\mathrm{P}_{\mathrm{i}}^{(0)}+\left(\mathrm{P}_{\mathrm{i}}^{(\mathrm{l})}-\mathrm{P}_{\mathrm{i}}^{(0)}\right) \times \mathrm{h}_{\mathrm{i}}, \quad \mathrm{h}_{\mathrm{i}} \in[0,1], \mathrm{i}=1,2,3, \cdots, \mathrm{d}
$$

Among them, $h_{i}$ is the proportional parameter; $d$ is the number of nodes in the link. Given a set of parameters $\left(h_{1}, h_{2}, h_{3}, \cdots, h_{d}\right)$, we can get a new path from start to finish, and the solution of ant colony algorithm is $\left(\mathrm{h}_{1}, \mathrm{~h}_{2}, \mathrm{~h}_{3}, \cdots, \mathrm{h}_{\mathrm{d}}\right)$. The division of the connection line is a fixed distance division method, and the division length is set to $\xi$, then the division number of each connection line $\mathrm{L}$ is the formula (5).

$$
\pi_{i}= \begin{cases}\ln t\left(\frac{L_{i}}{\xi}\right), & \text { if } \ln t\left(\frac{L_{i}}{\xi}\right) \text { is an even number } \\ \ln t\left(\frac{L_{i}}{\xi}\right)+1, & \text { if } \ln t\left(\frac{L_{i}}{\xi}\right) \text { is an odd number }\end{cases}
$$

\section{Improved Ant Colony Algorithm}

\subsection{Build the objective function}

In the actual intelligent transportation system, people need to take into account the influence of multiple factors on the route planning of travel. Not only the expected route is the shortest, but also the travel time and travel expenses should be taken into consideration. Ultimately, it seeks a comprehensive and optimal travel route. Therefore, the objective function $F_{k}$ is introduced as shown in formula (6).

$$
\left\{\begin{array}{l}
\mathrm{F}_{\mathrm{k}}=\mathrm{mD}_{\mathrm{k}}+\mathrm{nT}_{\mathrm{k}}+\mathrm{pM}_{\mathrm{k}} \\
\mathrm{M}_{\mathrm{k}}=\sigma \mathrm{D}_{\mathrm{k}}+\mathrm{C}
\end{array}\right.
$$

In the formula, m, n,p is the relative proportion of $D_{k}, T_{k}, M_{k}$ respectively; $D_{k}$ is the total length of the planned route; $T_{k}$ is the time required to go through the planned route; $\mathrm{M}_{k}$ is the cost required for the planned route; $\sigma$ and $\mathrm{C}$ are the fuel cost and the toll fee respectively for the unit route. Through the analysis of $\mathrm{F}_{\mathrm{k}}$, the optimal scheme of various influencing factors in travel is considered as the optimal planning path.

\subsection{Pheromone update}

Pheromone updates include real-time pheromone updates and path pheromone updates. The real-time pheromone update means that the pheromone of the node is updated after each node is selected, and the formula (7) can be used to indicate. $\tau_{0}$ is the pheromone initial value.

$$
\tau_{\mathrm{i}, \mathrm{j}}=(1-\rho) \tau_{\mathrm{i}, \mathrm{j}}+\rho \tau_{0}
$$

The path pheromone update is to select the optimal path after completing the search from the initial point to the end point, and to update the pheromone at each point on the path, which can be represented by equation (8).

$$
\tau_{\mathrm{i}, \mathrm{j}}=(1-\rho) \tau_{\mathrm{i}, \mathrm{j}}+\rho \Delta \tau_{\mathrm{i}, \mathrm{j}}
$$

Since the travel distance, time, cost and other factors need to be comprehensively investigated in the planning path, when each ant updates the pheromone of the node, the above three influencing factors need to be considered, and the pheromone increment contribution of all ants to the side $\mathrm{e}_{\mathrm{ij}}$ can be expressed by formula (9).

$$
\Delta \tau_{i j}^{k}(t, t+1)=\left\{\begin{array}{cl}
Q / F_{k} & , \text { if ant } k \text { arrives to city } j \text { from city } i \text { in this cycle } \\
0 & , \text { otherwise }
\end{array}\right.
$$

\subsection{Algorithm Implementation Steps}

Use the improved ant colony algorithm to study the intelligent traffic system and plan out the optimal path. The specific implementation steps of the algorithm are as follows: 
(1) Establish a spatial model and use the Dijkstra algorithm to generate the initial sub-optimal path. Finally complete the path planning initialization;

(2) On the basis of the initial path, used the improved ant colony algorithm to start the search and the pheromone is updated in real time;

(3) To determine whether the termination condition is satisfied. If it is satisfied, the optimal path is output. If the termination condition is not satisfied, the ant continues to search and repeat the execution step (2).

The algorithm flowchart is shown in Figure 1.

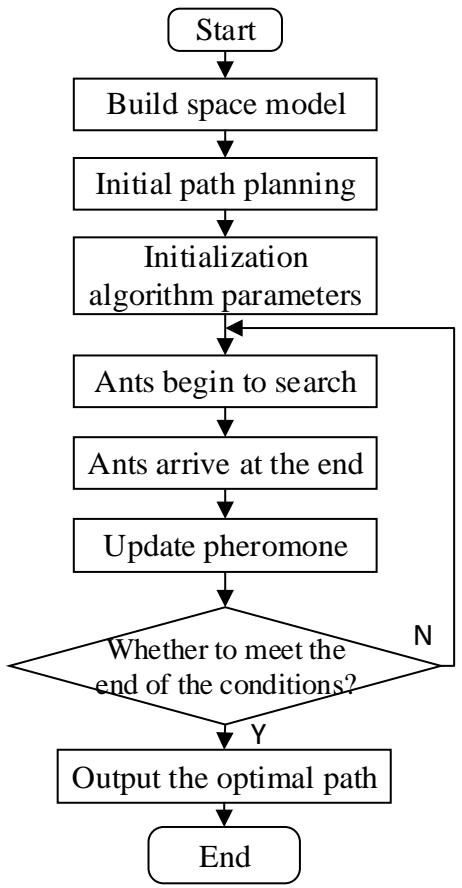

Fig. 1: The algorithm flowchart.

\section{Simulation Experiment and Result Analysis}

In order to verify the feasibility and effectiveness of the improved ant colony algorithm, this study designed a two-dimensional planning space which have obstacles. $\mathrm{S}$ is the beginning point, and $\mathrm{T}$ is the end point (as shown in Figure 2). Using Matlab2012a software and the improved ant colony algorithm, the optimal planning path is solved.

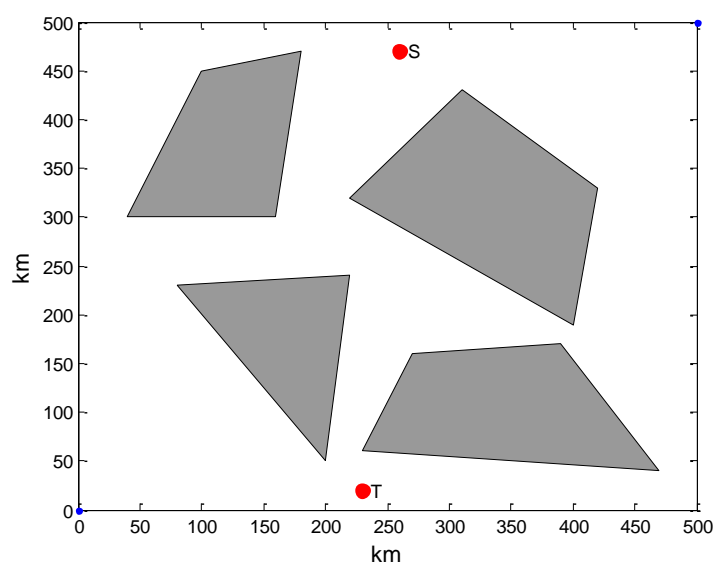

Fig. 2: A two-dimensional planning space.

First MAKLINK graph algorithm is used to establish the path planning of the two-dimensional space. Then with the Dijkstra algorithm planning out an initial path from start to finish (as shown in Figure 3, the blue line indicates the initial path). And the initialization algorithm parameters are initialized, and the related parameters of initialization are shown in Table 1. 


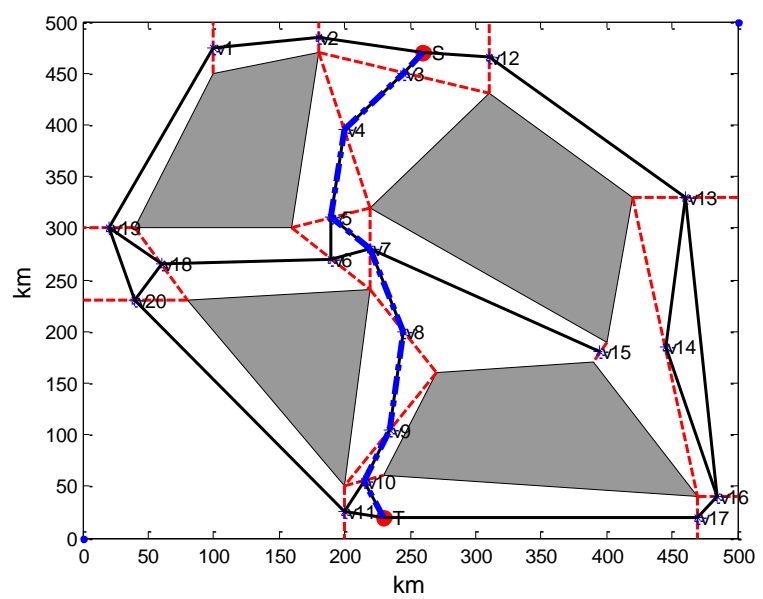

Fig. 3: An initial path.

Table 1: Initialization parameters

\begin{tabular}{ccc}
\hline The serial number & Parameter & Parameter Values \\
\hline 1 & population size & 100 \\
2 & Maximum number of iterations & 800 \\
3 & Initial value of pheromone & 0.0003 \\
4 & $\rho$ & 0.1 \\
5 & $\alpha$ & 1 \\
6 & $\beta$ & 0.35 \\
\hline
\end{tabular}

Figure 4 shows the adaptability curve of the improved ant colony algorithm, which shows that the algorithm has a better robustness and better system convergence.

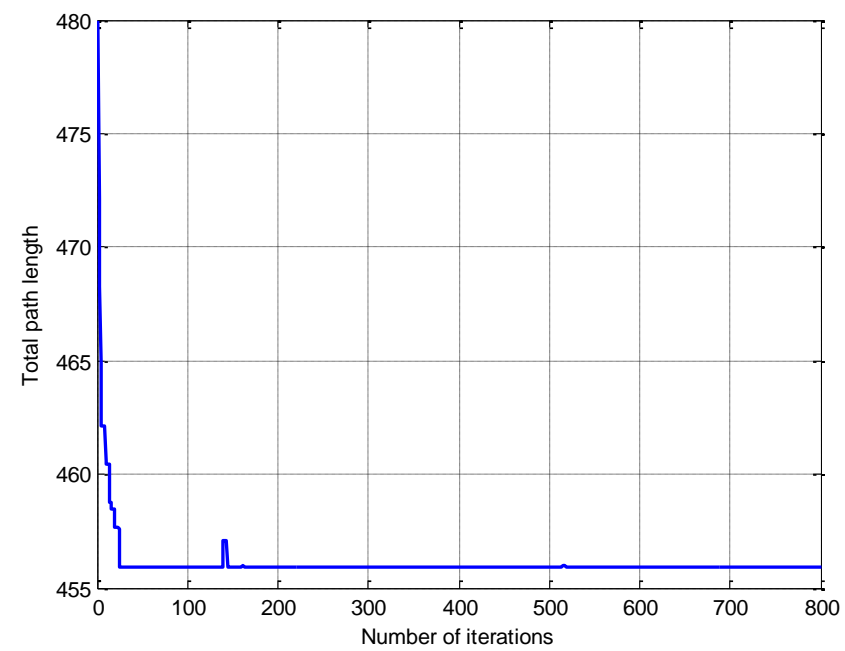

Fig. 4: The adaptability curve of the improved ant colony algorithm.

\section{Conclusion}

This paper aimed at the problems existing in the intelligent transportation system, combined the factors such as distance, time and cost of vehicle travel, improved on the basis of the basic ant colony algorithm, established the objective function, updated the pheromone, and finally completed the path to the moving vehicle and sought a comprehensive optimal driving path. This method improved the effectiveness and efficiency of path planning. But it cannot plan the path in real time when unexpected events happened, and also affected by the factors of path planning and road limit. So we need further research for finding a more reasonable path. 


\section{Acknowledgment}

This work was supported by the development of plant growth air purification plant (Project No.ZZJ0011403).

\section{References}

[1] X. Bai, Y. Wang. The Application of Ant Colony Algorithm in the Field of Intelligent Transportation. Electronic Technology and Software Engineering. 2016 (15): 181

[2] J.T. Teng, F.Z. Zhang, Y.P. Li, Y.Z. Fan. Research on the Genetic Algorithm of Vehicle Scheduling Problem in Intelligent Transportation System. Journal of Beihang University. 2003 (01): 13-16.

[3] S.J. Li, Y. Zhang, Y. Gong. Research on Intelligent Traffic Optimal Path Based on Ant Colony Algorithm. Journal of Changchun University of Science and Technology (Natural Science Edition). 2003 (01): 13-16.

[4] L. Tang, T.J. Fang. Path Planning Method Based on Improved Ant Colony Algorithm. Journal of China University of Science and Technologying. 2009, 39 (09): 980-983+995.

[5] H. Wang, L.B. Long, J.L. Wang, H.Y. Chen, X.J. Shao, Z.H. Zhu. Research on Path Planning of Parking System Based on Dijkstra-Ant Colony Algorithm. Journal of Engineering Design. 2016, 23 (05):489-496.

[6] H.B. Duan. Ant Colony Algorithm Principle and Application. Beijing: Science Press. 2005.

[7] F. Gao. MATLAB Intelligent Algorithm Super Study Manual. People Post Press. 2014. 